D. Portelli and W. Spangher

Nagoya Math. J.

Vol. 110 (1988), 137-149

\title{
ON THE DIVISOR CLASS GROUPS OF A TWO-DIMENSIONAL LOCAL RING AND ITS FORM RING
}

\author{
DARIO PORTELLI AND WALTER SPANGHER
}

\section{Introduction}

Let $A$ be a noetherian ring and let $I$ be an ideal of $A$ contained in the Jacobson radical of $A$ : $\operatorname{Rad}(A)$. We assume that the form ring of $A$ with respect to the ideal $I: G=\operatorname{Gr}(A, I)$, is a normal integral domain. Hence $A$ is a normal integral domain and one can ask for the links between $\mathrm{Cl}(A)$ and $\mathrm{Cl}(G)$.

Let $R=\oplus_{n \in Z} I^{n}$ be the Rees algebra of $A$ with respect to the ideal $I$ (see $\S 2$ ). In a previous paper [20], the authors have proved that $\mathrm{Cl}(A)$ $\simeq \mathrm{Cl}(R)$; moreover there exists a "canonical" map $j: \mathrm{Cl}(R) \rightarrow \mathrm{Cl}(G)$ deduced from the hypersurface section $R \rightarrow G=R / u R(\S 1)$. Following the ideas of Lipman's paper [18], in [20] an attempt was made to find out sufficient conditions for $\operatorname{ker}(j)=0$, (resp.: for $\operatorname{ker}(j)$ to be a torsion group). But this sufficient conditions become almost tautological when $\operatorname{dim}(A)=2$ and $\operatorname{ht}(I)=2$ (i.e. when $A$ is a local ring and $I=\operatorname{Rad}(A)$; see $\S 1$ ). This paper deals with this last case.

The main result of the paper is Theorem 4; this theorem can be proved also by using the geometrical machinery of Grothendieck, Danilov, Boutot and Bădescu-Fiorentini [15, 8, 9, 6, 3] (see also the Remark 3 after the proof of Theorem 4).

Our proof mainly uses simple tools of Commutative Algebra and standard facts of Local Cohomology theory. A key point is the finiteness of a suitable local cohomology module which we derive from [15].

It is also interesting that the short exact sequences which appear in Theorem 1 of [18] are the same which appear in our proof. In a certain sense, this circumstance unifies the two techniques.

However the problem of the injectivity of $j: \mathrm{Cl}(R) \rightarrow \mathrm{Cl}(G)$ for a general hypersurface section $R \rightarrow G=R / u R, \operatorname{dim}(G)=2$, is rather different

Received September 26, 1986. 
from that considered in this paper as the example given in Section 3 shows.

$\S 1$.

In [10] Danilov has studied the links between the groups $\mathrm{Cl}(A[[T]])$ and $\mathrm{Cl}(A)$, where $A$ is a normal integral domain. To do this, he has defined a canonical map $j: \mathrm{Cl}(A[[T]]) \rightarrow \mathrm{Cl}(A)$. But in fact Danilov's definition works more generally to give a map $j: \mathrm{Cl}(R) \rightarrow \mathrm{Cl}(R / u R)$ for any normal integral domain $R$ and nonunit $u \in R$ such that $R / u R=G$ is also a normal integral domain. Let us recall the construction of $j$ from the viewpoint of this paper.

First of all, $\mathrm{Cl}(R)$ can be thought as the group of isomorphism classes of finitely generated, reflexive, rank one $R$-modules [18, 31]; a similar interpretation holds for $\mathrm{Cl}(G)$. Let $F$ be a finitely generated, reflexive, rank one $R$-module; we set: $[F]_{R}=$ (isomorphism class of $\left.F\right) \in \mathrm{Cl}(R)$. Let $E=F \otimes_{R} G$ with $F$ as above; then $E^{* *}=\operatorname{Hom}_{G}\left(\operatorname{Hom}_{G}(E, G), G\right)$ is a finitely generated, reflexive, rank one $G$-module. By this interpretation of the class group, we have, following [18]: $j\left([F]_{R}\right)=\left[E^{* *}\right]_{G}$.

From now on we assume that $R$ is a $Z$-graded ring, i.e. $R=\oplus_{n \in Z} R_{n}$, and $u \in R$ is a homogeneous element. Let $\xi \in \mathrm{Cl}(R)$; then $\xi=[\mathfrak{b}]_{R}$ for some homogeneous integral divisorial ideal $\mathfrak{b}$ of $R$. If $\mathfrak{b}=\mathfrak{b}^{\prime} \cap u^{n} R,(n \geqslant 0)$, where $\mathfrak{b}^{\prime}$ is a homogeneous divisorial ideal with $\mathfrak{b}^{\prime} \not \subset u R$; then $[\mathfrak{b}]_{R}=\left[\mathfrak{b}^{\prime}\right]_{R}$ since $u$ is a prime element of $R$. Therefore there is no loss of generality in assuming that $\mathfrak{b} \not \subset u R$, or equivalently that $u$ is regular for $R / \mathfrak{b}$. Then we get: $\mathfrak{a}=\mathfrak{b} \otimes_{R} G \simeq \mathfrak{b} / u \mathfrak{b} \simeq \mathfrak{b}+u R / u R$ and $j\left([\mathfrak{b}]_{R}\right)=\left[\left((\mathfrak{a})^{-1}\right)^{-1}\right]_{G}$ where $\left((\mathfrak{a})^{-1}\right)^{-1}$ denotes, as usual, the divisorial ideal associated to $\mathfrak{a}$. In the sequel we will always refer to this simpler setup whenever the map $j$ is concerned.

The homomorphism $j$ ties together the groups $\mathrm{Cl}(R)$ and $\mathrm{Cl}(G)$. In particular one can ask the following questions for $j$ : when is $j$ surjective? and: when is $j$ injective?

The following proposition, concerning the latter question, has been proved in [20] following the general ideas of Lipman's paper [18]:

Let $R=\oplus_{n \in Z} R_{n}$ be a Z-graded normal integral domain and let $u \in h$ $\operatorname{Rad}(R)$ be a non-zero homogeneous element such that $G=R / u R$ is also a normal domain. Suppose that the canonical map $j_{Q}: \mathrm{Cl}\left(R_{Q}\right) \rightarrow \mathrm{Cl}\left(G_{P}\right)$ is injective (resp.: $\operatorname{ker}\left(j_{Q}\right)$ is a torsion group) for every homogeneous prime ideal $Q$ of $R$ such that: $u \in Q$ and depth $\left(R_{Q}\right) \leqslant 3$ (of course $P=Q / u R$ ). Then also $j: \mathrm{Cl}(R) \rightarrow \mathrm{Cl}(G)$ is an injective map (resp.: $\operatorname{ker}(j)$ is a torsion 
group).

Let us observe that the hypotheses of this proposition forces $\operatorname{dim}(G)$ $\geqslant 2$. But if $G$ is a normal integral domain and $\operatorname{dim}(G)=2$, then $G$ is a C.M. ring. Hence also $R$ is a C.M. ring ( $u \in h-\operatorname{Rad}(R)$; see [7], Proposition 2.2) and the above proposition becomes almost tautological. If $\operatorname{dim}(G)$ $\leqslant 1, \mathrm{Cl}(R)$ is simple to compute.

Therefore only the case $\operatorname{dim}(G)=2$ remains still open. After all, this is not so surprising; in fact the case $\operatorname{dim}(A)=2$ was the hardest to solve also for the problem of Danilov-Samuel, i.e. for the hypersurface section $A[[T]] \rightarrow A$ (see $[26,25,28,9])$. Essentially, there are two (non tautological) ways to handle the case of a general hypersurface section $R \rightarrow G$ when $\operatorname{dim}(G)=2$. The most recent one is due to Flenner (see Lemma 3.4 of [12]) and is inspired to Theorem 1 of [18]. The other one is used in this paper and comes from Hilfsatz 3 of [28], or Remarque p. 164 of [27]; it is summarized in the following proposition:

Proposition 1. Let $R=\oplus_{n \in Z} R_{n}$ be a Z-graded, normal integral domain and let $u \in h-\operatorname{Rad}(R)$ be a non-zero, homogeneous element such that $G=R / u R$ is also a normal integral domain. Assume that $G$ is a C.M. ring. Let $\xi \in \mathrm{Cl}(R)$ and let $\mathfrak{b} \subset R$ be a homogeneous, proper, divisorial ideal such that $\xi=[\mathfrak{b}]_{R}, \mathfrak{b} \not \subset u R$ and $\mathfrak{a}^{-1}$ is a h-free G-module, where $\mathfrak{a}=$ $\mathfrak{b} \otimes_{R} G$. Then $\xi=0$, i.e. $\mathfrak{b}$ is $h$-free, if and only if $R / \mathfrak{b}$ is a C.M. ring.

Proof. Let $R / \mathfrak{b}$ be a C.M. ring; since $u$ is regular for $R / \mathfrak{b}$, the ideal $\mathfrak{b}+u R / \mathfrak{b}$ is an unmixed ideal of height one of $R / \mathfrak{b}$. Therefore $\mathfrak{b}+u R$ is an unmixed ideal of height two of $R$, hence $\mathfrak{a}=\mathfrak{b}+u R / u R$ is an unmixed ideal of height one of $G$. It follows that $\mathfrak{a}=\left((\mathfrak{a})^{-1}\right)^{-1}$, so $\mathfrak{a}$ is $h$-free and then $\mathfrak{b}$ is $h$-free (see [5], Ch. II, 3.2, Proposition 5; with suitable modifications to the homogeneous case). The converse is trivial because $R$ is a C.M. ring.

\section{$\S 2$.}

Let $A$ be a ring and $I \subseteq \operatorname{Rad}(A)$ an ideal of $A$. We fix the following notation: $R=R(A, I)=\oplus_{n \in Z} I^{n}\left(I^{n}=A\right.$ for $\left.n \leqslant 0\right)$ is the Rees algebra of $A$ with respect to $I$. If $T$ is an indeterminate over $A$, let $u=T^{-1}$. We have $R=A\left[a_{1} T, \cdots, a_{r} T, u\right] \subseteq A[T, u]$ where $I=\left(a_{1}, \cdots, a_{r}\right) . G=\operatorname{Gr}(A, I)$ $=\oplus_{n \geq 0} I^{n} / I^{n+1}$ is the form ring of $A$ with respect to $I$; the irrelevant ideal of $G$ is $G_{+}=\oplus_{n>0} I^{n} / I^{n+1}$; let $\mathfrak{a}^{*}=\mathfrak{a} \cdot A[T, u] \cap R$ where $\mathfrak{a}$ is an ideal of 
$A ; \mathfrak{a}^{*}$ is a graded ideal of $R ; \operatorname{In}_{I}(\mathfrak{a})$ is the graded ideal of $G$ generated by the initial forms $\operatorname{In}(x)$ for all $x \in \mathfrak{a}$.

We refer to $[7,20,22,24]$ for the general properties of these rings and ideals. However, for the sake of completeness, let us recall the following ones: first $G \simeq R / u R ; u$ is a homogeneous element and $\operatorname{deg}(u)=-1$. Moreover $u \in h-\operatorname{Rad}(R)$ and, finally, $\operatorname{In}_{I}(\mathfrak{a})=\mathfrak{a}^{*}+u R / u R$. If $G$ is a normal integral domain, then also $R$ and $A$ are normal integral domains. If $G$ is a normal integral domain we can consider the map $j: \mathrm{Cl}(R) \rightarrow \mathrm{Cl}(G)$ defined in Section 1. Moreover, since $u$ is a prime element of $R$ it is easy to see that $\mathrm{Cl}(A) \underset{\psi}{\stackrel{\sim}{\longrightarrow}} \mathrm{Cl}(R)$ (see [20], Proposition 1); to be precise, the isomorphism $\psi$ between $\mathrm{Cl}(A)$ and $\mathrm{Cl}(R)$ is given by $\psi\left([\mathfrak{a}]_{A}\right)=\left[\mathfrak{a}^{*}\right]_{R}$, where $\mathfrak{a}$ is an integral, divisorial ideal of $A$. Therefore, by composition, we get a homomorphism $i: \mathrm{Cl}(A) \rightarrow \mathrm{Cl}(G)$ such that $i\left([\mathfrak{a}]_{A}\right)=\left[\left(\left(\operatorname{In}_{I}(\mathfrak{a})\right)^{-1}\right)^{-1}\right]_{G}$ where $\mathfrak{a}$ is as above.

We begin the study of the map $j: \mathrm{Cl}(R(A, I)) \rightarrow \mathrm{Cl}(G(A, I))$ with a statement concerning the surjectivity of $j$.

Proposition 2. Let $(A, m)$ be a local, henselian ring with $\operatorname{dim}(A)=2$. Suppose that $G=\operatorname{Gr}(A, m)$ is a normal integral domain. Then the map $j: \mathrm{Cl}(R) \rightarrow \mathrm{Cl}(G)$ is surjective.

Proof. Let $P$ be a homogeneous, height one, prime ideal of $G$. Pick $\bar{x} \in P-P^{(2)}$ with $\bar{x}$ homogeneous. Let $x \in A$ be an element such that $\operatorname{In}(x)=\bar{x}$. Expand $x A$ to a prime ideal $Q$, maximal among those disjointed from the multiplicatively closed set $\{y \in A \mid \operatorname{In}(y) \notin P\}$; clearly ht $(Q)=1$. From the isomorphism $G(A / Q, m / Q) \simeq G(A, m) / \operatorname{In}(Q)$ and the choice of $\bar{x}$ it follows that $\left((\operatorname{In}(Q))^{-1}\right)^{-1}=P$ (see Lemma 6 of [1]). Therefore $j\left(\left[Q^{*}\right]_{R}\right)=$ $[P]_{G}$, where $Q^{*}=Q \cdot A[T, u] \cap R$. Since $\mathrm{Cl}(G)$ is generated by the classes of homogeneous, height one prime ideals of $G$, the thesis follows.

Remark. The following example shows that we cannot delete the requirement " $G$ is normal" in Proposition 2. Let $A=R[[X, Y, Z]] /\left(x^{2}+\right.$ $\left.Y^{2}+Z^{3}\right)$ ( $R$ is the field of real numbers); $A$ is a local complete factorial ring, and $\operatorname{dim}(A)=2$ (see ex. $(25,4)$ of $[17])$. But $G(A, m) \simeq R[X, Y, Z] /$ $\left(X^{2}+Y^{2}\right)$ is not even normal, hence it cannot be factorial.

The next proposition deals with the case $h t(I) \leqslant 1$.

Proposition 3. Let $A$ be a ring with $\operatorname{dim}(A)=2$, and let $I \subset \operatorname{Rad}(A)$ be an ideal of $A$ such that ht $(I) \leqslant 1$ and $G=\operatorname{Gr}(A, I)$ is a normal integral 
domain.

a) If I is invertible, then $\mathrm{Cl}(A)$ is embedded in $\mathrm{Cl}(G)$;

b) If $G$ is an almost factorial ring (in particular if $G$ is a factorial ring) then $I$ is invertible.

Proof. If ht $(I)=0$ there is nothing to prove, so we assume that ht $(I)=1$. We have that $G_{0}=A / I$ is a Krull domain. Since $\operatorname{dim}(A / I)$ $=1, A / I$ is a Dedekind domain; in particular it satisfies the property $\left(R_{1}\right)$ of Serre and moreover $\mathrm{Cl}(A / I) \simeq \operatorname{Pic}(A / I)$. But $I$ is invertible and $I \subseteq \operatorname{Rad}(A)$; by localization at the maximal ideals, we have that $A$ is an $\left(R_{2}\right)$ ring, hence $A$ is locally factorial and $\mathrm{Cl}(A) \simeq \operatorname{Pic}(A)$. Since $I \subseteq$ $\operatorname{Rad}(A)$, the canonical map $\operatorname{Pic}(A) \rightarrow \operatorname{Pic}(A / I)$ is injective (see [2], Proposition 1.4). From the hypothesis " $I$ is invertible" it follows that $G$ is a flat $G_{0}$-module (Lemma 2.1 of [23]). So the extension $G_{0} \rightarrow G$ satisfies condition (PDE) and the induced homomorphism $\mathrm{Cl}\left(G_{0}\right) \rightarrow \mathrm{Cl}(G)$ is injective ([13], Proposition 10.7). This completes the proof of $a$ ).

The irrelevant ideal $G_{+}$of $G$ is a prime ideal. We easily gat $G_{+}^{(p)}=$ $\oplus_{n \geqslant p} G_{n}=G_{+}^{p}$ for all $p>0$. Therefore, since $G_{+}=\operatorname{In}(I)$ and $\mathrm{ht}(\operatorname{In}(I))=$ ht $(I)=1$, we have that $G_{+}$is a projective $G$-module, since $G$ is an almost factorial ring. But $I / I^{2} \simeq G_{+} / G_{+}^{2} \simeq G_{+} \otimes_{G} G_{0}$, so $I / I^{2}$ is a projective $A / I$ module. Then $I$ is locally principal since $A_{I}$ is a DVR (see Lemma 2.1 of [23]).

Remark. In general the embedding $f: \mathrm{Cl}(A) \rightarrow \mathrm{Cl}(G)$ constructed in the proof is different from the map obtained by the composition of the isomorphism $\psi: \mathrm{Cl}(A) \stackrel{\Im}{\rightarrow} \mathrm{Cl}(R)$ and $j: \mathrm{Cl}(R) \rightarrow \mathrm{Cl}(G)$, i.e. from the map $i$.

If $\operatorname{ht}(I)=2$, from $I \subseteq \operatorname{Rad}(A)$ and $\operatorname{dim}(A)=2$ it follows that $(A, I)$ is a local ring, i.e. $I=\operatorname{Rad}(A)$. In the next theorem a sufficient condition is given for the map $j: \mathrm{Cl}(R) \rightarrow \mathrm{Cl}(G)$ to be injective when $\mathrm{ht}(I)=2$.

Local cohomology is the key tool in the proof of Theorem 4, so let us make some general remarks on it. Let $S$ be a graded ring, $J \subset S$ a graded ideal and $M$ a graded $S$-module. Since $H_{J}^{i}(M)=\varliminf_{n} \operatorname{Ext}_{S}^{i}\left(S / J^{n}, M\right)$ for all $i \geqslant 0$, the local cohomology modules are graded modules in this case. Moreover, let

$$
0 \longrightarrow M^{\prime} \underset{(d)}{\stackrel{\rho}{\longrightarrow}} M \underset{(t)}{\stackrel{\pi}{\longrightarrow}} M^{\prime \prime} \longrightarrow 0
$$

be a short exact sequence of graded $S$-modules. Suppose $\rho$ and $\pi$ graded, 
respectively of degree $d$ and $t$. Then the corresponding long exact cohomology sequence is graded as follows:

$$
\cdots \longrightarrow H_{J}^{i}\left(M^{\prime}\right) \underset{(d)}{\stackrel{H_{J}^{i}(\rho)}{\longrightarrow}} H_{J}^{i}(M) \stackrel{H_{J}^{i}(\pi)}{(t)} H_{J}^{i}\left(M^{\prime \prime}\right) \frac{\theta_{i}}{(-d-t)} H_{J}^{i+1}\left(M^{\prime}\right) \longrightarrow \cdots
$$

Theorem 4. Let $(A, Q)$ be a local ring with $\operatorname{dim}(A)=2$. Assume that:

$$
\left(H_{G+}^{2}(G)\right)_{n}=0 \text { for all } n>0
$$

Then the map $\mathrm{j}: \mathrm{Cl}(R) \longrightarrow \mathrm{Cl}(G)$ is injective.

Proof. We shall give the proof in several steps.

Step 1. Let $\mathfrak{b}$ be a homogeneous integral (proper) divisorial ideal of $R$ such that $\mathfrak{b} \not \subset u R$; suppose that $\mathfrak{a}^{-1}$ is a $h$-free $G$-module (where a denotes, as usual, $\left.\mathfrak{b} \otimes_{R} G \simeq \mathfrak{b}+u R / u R\right)$. By Proposition 1 we have only to show that $R / \mathfrak{b}$ is a C.M. ring. $R$ is a $h$-local ring; indeed $\mathfrak{m}=\left(Q^{*}, u\right)$ is a maximal ideal of $R$ and $\mathfrak{m}=h-\operatorname{Rad}(R)$. Then also $R / \mathfrak{b}$ is a $h$-local ring and $\mathfrak{n}=\mathfrak{m} / \mathfrak{b}=h-\operatorname{Rad}(R / \mathfrak{b}) . \quad R / \mathfrak{b}$ is a C.M. ring if and only if $(R / \mathfrak{b})_{\mathfrak{n}}$ is a C.M. ring (see [19], Theorem 1.1). But $(R / \mathfrak{b})_{\mathfrak{n}}$ is a C.M. ring if and only if $H_{\overline{\mathfrak{n}}}^{0}\left((R / \mathfrak{b})_{\mathfrak{n}}\right)=H_{\overline{\mathfrak{n}}}^{1}\left((R / \mathfrak{b})_{\mathfrak{n}}\right)=0 \quad$ (where $\left.\overline{\mathfrak{n}}=\mathfrak{n} \cdot(R / \mathfrak{b})_{\mathfrak{n}}\right)$. Now $H_{\overline{\mathfrak{n}}}^{0}\left((R / \mathfrak{b})_{\mathfrak{n}}\right)$ $=0$ since $u$ is a regular element for $R / \mathfrak{b}$. $R$ is a C.M. ring and $\operatorname{depth}\left(R_{\mathrm{m}}\right)$ $=3$; then from the long exact sequence for the local cohomology and from Theorem 4.3 of [29] we get: $H_{\mathrm{n}}^{1}(R / \mathfrak{b}) \simeq H_{\mathrm{m}}^{1}(R / \mathfrak{b}) \simeq H_{\mathrm{m}}^{2}(\mathfrak{b})$. As $H_{\mathrm{n}}^{1}(R / \mathfrak{b})$ $\otimes_{R / \mathfrak{b}}(R / \mathfrak{b})_{\mathfrak{n}} \simeq H_{\mathfrak{n}}\left((R / \mathfrak{b})_{\mathfrak{n}}\right)$ (see [29], Theorem 5.1), it will be sufficient to show that $H_{\mathrm{m}}^{2}(\mathfrak{b})=0$.

Step 2. $H_{\mathrm{m}}^{2}(\mathfrak{b})$ is a finitely generated $R$-module. To see this it is sufficient to prove that $H_{\hat{\mathrm{m}}}^{2}(\hat{\mathfrak{b}})$ is a finitely generated $\hat{R}$-module, where $\hat{R}=(\widehat{R, \mathfrak{m}}) \simeq\left(\widehat{R_{\mathrm{m}}, \mathfrak{m} R_{\mathrm{m}}}\right)$. In fact we have (see [30], Theorem 4.5): $H_{\hat{\mathrm{m}}}^{2}(\hat{\mathrm{b}})$ $\simeq H_{\mathrm{m} R_{\mathrm{m}}}^{2}\left(\mathfrak{G} R_{\mathrm{m}}\right) \otimes_{R_{\mathrm{m}}} \hat{R}$; therefore $H_{\hat{\mathrm{m}}}^{2}(\hat{\mathrm{b}})$ is finitely generated over $\hat{R}$ if and only if $H_{\mathrm{m} R_{\mathrm{m}}}^{2}\left(\mathfrak{b} R_{\mathrm{m}}\right)$ is a finitely generated $R_{\mathrm{m}}$-module (see [5], Proposition 11, Ch. I. 3.6.) and this last condition is equivalent to " $H_{\mathrm{m}}^{2}(\mathfrak{b})$ is finitely generated over $R$ " since $H_{\mathrm{m} R_{\mathrm{m}}}^{2}\left(\mathfrak{b} R_{\mathrm{m}}\right) \simeq H_{\mathrm{m}}^{2}(\mathfrak{G}) \otimes_{R} R_{\mathrm{m}}$ (see [21], Proposition 11). Since $\hat{R}$ is a C.M. ring and since $\hat{b}$ is an unmixed ideal of height one of $\hat{R}$ (see [14], 9.3 and 13.8), for every prime ideal $P$ of $\hat{R}$ such that $h t(P)=2$, $\operatorname{depth}\left(\hat{\mathfrak{b}}_{P}\right)=2$. The finite generation of $H_{\hat{\mathrm{m}}}^{2}(\hat{\mathrm{b}})$ over $\hat{R}$ then follows from [15], Expose VIII, Corollaire 2.3. 
Step 3. The hypothesis " $a^{-1}$ is $h$-free" implies $\left((a)^{-1}\right)^{-1}=x G$, where $x$ is a homogeneous element of $G$ of degree $d>0$. Then $\mathfrak{a}=\mathfrak{b}+u R / u R$ $=x G \cap I$, where $I$ is an eventual embedded primary component; since $a$ is homogeneous and $\operatorname{dim}(G)=2, I$ is irrelevent, i.e. $\sqrt{I}=G_{+}$. Now we have $H_{G_{+}}^{1}(G)=H_{G_{+}}^{0}(G)=0$ since $G$ is a C.M. ring. But $\left((\mathfrak{a})^{-1}\right)^{-1}=x G \simeq$ $G(-d)$, hence $H_{G_{+}}^{i}\left(\left((\mathfrak{a})^{-1}\right)^{-1}\right) \simeq H_{G_{+}}^{i}(G)(-d)$. From the short exact sequence:

$$
0 \longrightarrow a \longrightarrow\left((a)^{-1}\right)^{-1} \longrightarrow C \longrightarrow 0
$$

it follows that $H_{G_{+}}^{1}(\mathfrak{a}) \simeq H_{G_{+}}^{0}(C)=C$ where the isomorphism is of degree zero. Then from $\left(\left((\mathfrak{a})^{-1}\right)^{-1}\right)_{n}=0$ for all $n<d$ we get: $\left(H_{G_{+}}^{1}(\mathfrak{a})\right)_{n}=0$ for all $n<d$. Since $\operatorname{Supp}(C) \subseteq\left\{G_{+}\right\}$we have $H_{G_{+}}^{i}(C)=0$ for all $i>0$. Therefore from the long exact cohomology sequence associated to (2) we get: $H_{G_{+}}^{2}(\mathfrak{a}) \simeq H_{G_{+}}^{2}\left(\left((\mathfrak{a})^{-1}\right)^{-1}\right) \simeq H_{G_{+}}^{2}(G)(-d)$ where both isomorphisms are of degree zero. Now the hypothesis (1) comes into play to get: $\left(H_{G_{+}}^{2}(\mathfrak{a})\right)_{n}=0$ for all $n>d$.

Finally, from the canonical isomorphisms (of degree zero) $H_{G_{+}}^{1}(\mathfrak{a}) \simeq$ $H_{\mathrm{m}}^{1}(\mathfrak{a})$ and $H_{G_{+}}^{2}(\mathfrak{a}) \simeq H_{\mathrm{m}}^{2}(\mathfrak{a})$ we get:

$$
\begin{array}{ll}
\left(H_{\mathrm{m}}^{1}(\mathfrak{a})\right)_{n}=0 & \text { for all } n<d, \\
\left(H_{\mathrm{m}}^{2}(\mathfrak{a})\right)_{n}=0 & \text { for all } n>d .
\end{array}
$$

Step 4. Let

$$
\cdots \longrightarrow\left(H_{\mathrm{m}}^{1}(\mathfrak{a})\right)_{n} \longrightarrow\left(H_{\mathrm{m}}^{2}(\mathfrak{G})\right)_{n+1} \stackrel{\cdot u}{\longrightarrow}\left(H_{\mathrm{m}}^{2}(\mathfrak{b})\right)_{n} \longrightarrow\left(H_{\mathrm{m}}^{2}(\mathfrak{a})\right)_{n} \longrightarrow \cdots
$$

be the long exact cohomology sequence corresponding to the short exact sequence:

$$
0 \longrightarrow \mathfrak{b} \frac{\cdot u}{(-1)} \mathfrak{b} \underset{(0)}{\longrightarrow} \mathfrak{a} \longrightarrow 0
$$

From (5) and (3) it follows that $u$ is a regular element for all homogeneous elements of $H_{\mathrm{m}}^{2}(\mathfrak{b})$ of degree $\leqslant d$. Let $x \in\left(H_{\mathrm{m}}^{2}(\mathfrak{b})\right)_{n}$ with $n \leqslant d$; by definition of local cohomology there exists a positive integer $t$ such that $\mathfrak{m}^{t} \cdot x=0$; but $u \in \mathfrak{m}$, hence $u^{t} \cdot x=0$; therefore $x=0$ and $\left(H_{\mathrm{m}}^{2}(\mathfrak{b})\right)_{n}=0$ for all $n \leqslant d$. (this is essentially the proof of Lemma 1.2 of [30]). Therefore, from (5) and (4) it follows $H_{\mathrm{m}}^{2}(\mathfrak{b})=u H_{\mathrm{m}}^{2}(\mathfrak{b})$. But $u \in h-\operatorname{Rad}(R)$, hence $H_{\mathrm{m}}^{2}(\mathfrak{b})=0$ by Step 2 and the homogeneous Nakayama's lemma.

\section{Remarks.}

1) Since $\hat{R}$ is a local ring we can derive the finite generation of 
$H_{\hat{\mathrm{m}}}^{2}(\hat{\mathfrak{b}})$ over $\hat{R}$, also from [15], Exposé $V$, Corollaire 3.6. Since $\hat{R}$ is flat over $R, u$ is a regular element for $\hat{R}$. Therefore: $\operatorname{Gr}(\hat{R}, u \hat{R}) \simeq \hat{R} / u \hat{R}[T] \simeq$ $\hat{G}[T]$, where $T$ is an indeterminate over $\hat{R} / u \hat{R}$ and $\hat{G}=\left(\widehat{G, G_{+}}\right)$. It follows that $\hat{R}$ is a normal integral domain (see $\S 3$, Proposition 6 , a)).

2) With the same notations of Theorem 4, but without the hypothesis (1), we can prove the following result:

For every ideal $J$ of $A$, let $\mathfrak{c}=J A[T, u] \cap R$. Then $\left(H_{\mathfrak{m}}^{2}(\mathfrak{c})\right)_{n}=0$ for all $n \leqslant 0$. Let $\{u, x, y\}$ be a homogeneous system of parameters in $R$ with $\operatorname{deg}(x)=\operatorname{deg}(y)=1$; thus the Čech complex of $c$ is given by:

$$
C^{\cdot}(u, x, y ; \mathfrak{c}) ; 0 \stackrel{d_{0}}{\longrightarrow} \mathfrak{c}_{u} \oplus \mathfrak{c}_{x} \oplus \mathfrak{c}_{y} \stackrel{d_{1}}{\longrightarrow} \mathfrak{c}_{u x} \oplus \mathfrak{c}_{u y} \oplus \mathfrak{c}_{x y} \stackrel{d_{2}}{\longrightarrow} \mathfrak{c}_{u x y} \longrightarrow 0
$$

All the modules have a natural grading and the maps $d_{i}$ are as usual. Assume $\sigma=\left(d /(u x)^{p} ; e /(u y)^{p} ; f /(x y)^{p}\right) \in \operatorname{ker}\left(d_{2}\right)$, (i.e. $-d y^{p}+e x^{p}-f u^{p}=0$ ) with $d, e, f$ homogeneous elements of $c$, and $\operatorname{deg}(d)=\operatorname{deg}(e)=\operatorname{deg}(f)-$ $2 p=n \leqslant 0$.

We prove that there exists $\rho=\left(a / u^{p} ; b / x^{p} ; c / y^{p}\right)$, with $a, b, c$ homogeneous elements of $c$, such that $d_{1}(\rho)=\sigma$ i.e. $a x^{p}-b u^{p}=d$ and $a y^{p}-$ $c u^{p}=e$ (the third equation $b y^{p}-c x^{p}=f$ is dependent upon the others). If $p \leqslant 0$, the proof is trivial. Let $p>0$. Since $\left(u^{p}, x^{p}, y^{p}\right)$ is an $R$-regular sequence, we have $d \in\left(x^{p}, u^{p}\right), e \in\left(y^{p}, u^{p}\right)$. On the other hand, one can easily prove that $\oplus_{n \leqslant 0}\left(x^{p}, u^{p}\right)_{n}$ and $\oplus_{n \leqslant 0}\left(y^{p}, u^{p}\right)_{n}$ are included in $\oplus_{n \leqslant 0}\left(u^{p}\right)_{n}$; hence $d, e \in\left(u^{p}\right)$.

We now recall that $u^{p}$ is regular for $R / c$; therefore the system

$$
\left\{\begin{array}{l}
a x^{p}-b u^{p}=d \\
a y^{p}-c u^{p}=e
\end{array}\right.
$$

has solutions if we take $a=0$.

3) Theorem 4 has many sources; in particular see $[8,12,3,4]$. Instead of condition (1) of Theorem 4 in these papers is used the equivalent condition:

$$
H^{1}\left(Y, \Theta_{Y}(n)\right)=0 \text { for all } n>0
$$

where $Y=\operatorname{Proj}(G)$ (see [16], Ch. III, Proposition 2.1.5.)

For the sake of completeness we briefly show how the geometrical techniques work to get results as in Theorem 4.

Let $\mathscr{R}=\oplus_{n \geqslant 0} Q^{n}$ be the blow-up algebra with respect to the ideal $Q$, and set: $X=\operatorname{Proj}(\mathscr{R}), Y=\operatorname{Proj}(G)$; let $\chi: Y \rightarrow X$ be the closed immersion 
deduced from the canonical map $\mathscr{R} \rightarrow \mathscr{R} / Q \mathscr{R} \simeq G$.

Since $A$ and $G$ are normal integral domains of dimension two, it follows easily that the canonical morphism $X \rightarrow \operatorname{Spec}(A)$ is a desingularization of $\operatorname{Spec}(A)$. In particular we get that the canonical morphism $\varphi: \operatorname{Pic}(X) \rightarrow \operatorname{Pic}(X-Y)$ is surjective (see [16] IV, 21. 6. 11). Moreover: $\operatorname{Ker} \varphi=\left[\Theta_{X}(1)\right] \cdot Z$, and this is an infinite cyclic group. But $X-Y \simeq$ $\operatorname{Spec}(A)-\{\mathfrak{m}\}$; therefore $\operatorname{Pic}(X-Y) \simeq \operatorname{Pic}(\operatorname{Spec}(A)-\{\mathfrak{m}\}) \simeq \mathrm{Cl}(A)$ (see $[13], 18.10)$ and we get the short exact sequence:

$$
0 \longrightarrow Z \longrightarrow \operatorname{Pic}(X) \longrightarrow \mathrm{Cl}(A) \longrightarrow 0
$$

Another well known short exact sequence is the following:

$$
0 \longrightarrow Z \longrightarrow \operatorname{Pic}(Y) \longrightarrow \mathrm{Cl}(G) \longrightarrow 0
$$

where the first morphism maps 1 to $\left[\Theta_{Y}(1)\right]$. Finally, we consider the morphism $\chi^{*}:$ Pic $(X) \rightarrow \operatorname{Pic}(Y)$ deduced from the closed immersion $\chi: Y$ $\hookrightarrow X$. Putting all together we get the following diagram:

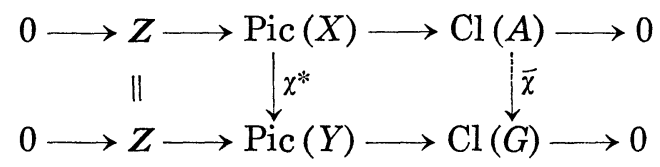

where $\bar{\chi}$ is deduced from $\chi^{*}$. It is easily checked that $\bar{\chi}=i$. From the "snake-lemma" it follows that the maps $\chi^{*}$ and $\bar{\chi}=i$ have isomorphic kernels and cokernels. The geometrical techniques developed in [15, 9, 6, 3] allow a direct study of $\chi^{*}$. Now we sketch their use.

First of all, we can define for all $n \geqslant 0$ a graded ring $G_{n}=$ $\oplus_{i \geqslant 0} Q^{i} / Q^{i+n+1}$; in particular we have $G_{0}=G$. If $m \geqslant n$ we get an epimorphic map $G_{m} \rightarrow G_{n}$. Let $Y_{n}=\operatorname{Proj}\left(G_{n}\right)$; we have a closed immersion $Y_{n} \rightleftarrows Y_{m}$ whenever $m \geqslant n$.

If $(A, Q)$ is henselian, then the sequence $\left\{\operatorname{Pic}\left(Y_{n}\right)\right\}_{n}$ is essentially constant and $\operatorname{Pic}(X)=\varliminf_{n}^{\lim } \operatorname{Pic}\left(Y_{n}\right)$ (see [6], Ch. IV, Proposition 6.2). By virtue of well-known Theorem of Mori ([13], Corollary 6.12), we can reduce to the case " $(A, Q)$ henselian" by replacing $A$ with $\hat{A}=(\widehat{A, Q})$. Moreover, for all $n \geqslant 0$ we have a short exact sequence of abelian sheaves on the topological space of $Y$ :

$$
0 \longrightarrow\left(i_{n+1}\right)_{*} \Theta_{Y}(n+1) \longrightarrow\left(\Theta_{Y_{n+1}}\right)^{*} \longrightarrow\left(\Theta_{Y_{n}}\right)^{*} \longrightarrow 1
$$

where $i_{n+1}: Y \rightarrow Y_{n+1}$ is the canonical closed immersion. Since $\operatorname{dim}(Y)=1$ 
the long exact sequence deduced from (7) is:

$$
H^{1}\left(Y, \Theta_{Y}(n+1)\right) \longrightarrow \operatorname{Pic}\left(Y_{n+1}\right) \longrightarrow \operatorname{Pic}\left(Y_{n}\right) \longrightarrow 0
$$

If condition (6) holds, we get $\chi^{*}: \operatorname{Pic}(X) \simeq \operatorname{Pic}(Y)$ and Theorem 4 follows. On the other hand, without any hypothesis on $G$, we get easily the following:

Proposition 5. If $\operatorname{char}(k)=p>0$, then $\operatorname{ker}\left(\chi^{*}\right)=\operatorname{ker}(i)$ is a $p$ torsion group.

With the same notations of Theorem 4, let $\mathfrak{b}$ be a homogeneous, proper, divisorial ideal of $R, \mathfrak{b} \not \subset u R$ such that $[\mathfrak{b}]_{R} \in \operatorname{ker}(j)$. If $\mathfrak{b}=P_{1}^{\left(n_{1}\right)}$ $\cap \cdots \cap P_{r}^{\left(n_{r}\right)}$ is the primary decomposition of $\mathfrak{b}$, put $\mathfrak{b}^{\left(p^{m}\right)}=P_{1}^{\left(n_{1} p^{m}\right)} \cap \cdots \cap$ $P_{r}^{\left(n_{r} p^{m}\right)}$ for $m>0$. Then Proposition 5 means that $H_{\mathrm{m}}^{2}\left(\mathfrak{b}^{\left(p^{m}\right)}\right)=0$ for some $m \geqslant 0$. The authors were unable to prove directly this fact.

\section{§3. Concluding remarks}

1) As the following counterexample shows, the hypothesis (1) of Theorem 4 does not suffice to deduce the injectivity of $j: \mathrm{Cl}(R) \rightarrow \mathrm{Cl}(G)$ when $R \rightarrow R / u R=G$ is a general hypersurface section. Let $k$ be an algebraically closed field. Let $R=k[X, Y, Z, W] /(X Y-Z W)=k[x, y, z, w]$ and let $G=R /(x-y) . \quad G$ is the homogeneous coordinate ring of a smooth conic in $\boldsymbol{P}_{k}^{2}$, hence $G$ satisfies the hypothesis (1) of Theorem 4 . But $\mathrm{Cl}(R)$ $\simeq Z$ and $\mathrm{Cl}(G) \simeq Z / 2 Z$.

2) If a form ring $G$ is given, we can consider two rings of special relevance for our problem: $G_{G_{+}}$and $\hat{G}=\left(\widehat{G, G_{+}}\right)$. This relevance is partially explained by the properties collected in the following:

Proposition 6. Let $G=G(A, I)$ be a normal integral domain, where $I \subseteq \operatorname{Rad}(A)$ as always. Then we have:

a) $\operatorname{Gr}\left(\hat{G}, \hat{G}_{+}\right) \simeq \operatorname{Gr}\left(G, G_{+}\right) \simeq G$, hence $\hat{G}$ is a normal integral domain.

b) Let $\mathfrak{c}$ be a homogeneous ideal of $G$; then $\operatorname{In}_{\hat{G}_{+}}(\mathfrak{c} \hat{G}) \simeq \mathfrak{c}$ where the isomorphism is that of a). In particular, let $m: \mathrm{Cl}(G) \rightarrow \mathrm{Cl}(\hat{G})$ the homomorphism deduced from the flat extension $G \rightarrow \hat{G}$, i.e. $m\left([\mathrm{c}]_{G}\right)=[\mathrm{c} \hat{G}]_{\hat{G}}$ for every integral divisorial ideal $\mathfrak{c}$ of $G$. Then $i \cdot m=1_{\mathrm{CI}(G)}$.

c) $\operatorname{Gr}\left(G_{G_{+}}, G_{+} G_{G_{+}}\right) \simeq G \otimes_{G / G+} K$ (graded isomorphism) where $K$ is the quotient field of $A / I$. In particular, if $I$ is maximal, then $G$ is a h-local ring and $\operatorname{Gr}\left(G_{G_{+}}, G_{+} G_{G_{+}}\right) \simeq G$.

d) Let $\mathfrak{c}$ be a homogeneous ideal of $G$; then $\operatorname{In}_{G_{+} G_{G_{+}}}\left(\mathfrak{c}_{G_{+}}\right)$is graded iso- 
morphic to $\mathfrak{c} \otimes_{G / G_{+}} K$, where $K$ is the residue field of $G_{G_{+}}$. In particular, if $I$ is maximal, we have $\operatorname{In}_{G_{+} G_{G+}}\left(\mathfrak{c}_{G_{+}}\right) \simeq \mathfrak{c}$; if we consider $\mathrm{Cl}(G) \stackrel{\sigma}{\longrightarrow} \mathrm{Cl}\left(G_{G_{+}}\right)$ $\stackrel{i}{\longrightarrow} \mathrm{Cl}(G)$ where $\sigma$ is the canonical isomorphism ([13], Corollary 10.3), we have $\bar{i} \cdot \sigma=1_{\mathrm{Cl}(G)}$ and consequently $\bar{i}=\sigma^{-1}$.

Proof. Easy calculations.

Now let $G=\oplus_{n \geqslant 0} G_{n}$ be a graded two dimensional normal domain such that $G_{0}$ is a field, $G=G_{0}\left[G_{1}\right]$ and $G$ is a finitely generated algebra over $G_{0}$. Since Danilov's condition DCG (i.e. $\mathrm{Cl}(G) \simeq \mathrm{Cl}(G[[T]])$ is equivalent to $\left(H_{G_{+}}^{1}(G)\right)_{n}=0$ for all $n \geqslant 0$ (see [12], Satz 4.4), from DCG condition it trivially follows that $\mathrm{Cl}(G) \simeq \mathrm{Cl}(\hat{G})$, since this is equivalent to $\left(H_{G_{+}}^{1}(G)\right)_{n}=0$ for all $n>0$. (see [12], Theorem 4.1). Moreover there exist factorial graded rings as $G$ such that $\mathrm{Cl}(G) \simeq \mathrm{Cl}(\hat{G})$ but not satisfying the DCG condition (see [9], page 128).

However, condition (1) is not necessary for $j$ to be injective as the following example shows.

Let $G=\boldsymbol{Q}[X, Y, Z] /\left(X^{4}+Y^{4}-Z^{4}\right)$ where $\boldsymbol{Q}$ is the fie]d of rational numbers; $\mathrm{Cl}(G)$ is finite (see [11]); but $\mathrm{Cl}(G) \neq \mathrm{Cl}(\hat{G})$, since $\mathrm{Cl}(\hat{G}) \simeq$ $\mathrm{Cl}(G) \oplus \boldsymbol{Q}$ (see [12]); then take $A=G_{G_{+}}$. Since $\bar{i}$ is an isomorphism (see Proposition 6.d)), $j$ is surjective by definition of $\bar{i}$. Morevoer $\mathrm{Cl}(R), \mathrm{Cl}(A)$ and $\mathrm{Cl}(G)$ are finite sets with the same number of elements; hence $j$ is injective.

3) The authors do not know the existence of factorial graded ring $G$ satisfying the general above-mentioned hypotheses and such that $\mathrm{Cl}(G) \not \mathrm{Cl}(\hat{G})$.

Acknowledgment. The authors are thankful to the referee for his suggestions.

\section{REFERENCES}

[1] S. S. Abhyankar, Nonprefactorial local ring, Amer. J. Math., 89 (1967), 1137-1146.

[2] M. Arezzo e S. Greco, Sul gruppo delle classi di ideali, Ann. Sc. Norm. Super. Pisa Cl. Sci. IV Ser., 21 (1967), 459-483.

[3] L. Bădescu e M. Fiorentini, Criteri di semifattorialità e di fattorialità per gli anelli locali con applicazioni geometriche, Ann. Mat. Pura Appl. IV Ser., 103 (1975), 211-222.

[ 4 ] J. Bingener und U. Storch, Zur berechnung der Divisorenklassengruppen kompletter lokaler Ringe, Nova Acta Leopold. Neue Folge, 52/240 (1981), 7-63.

[5] N. Bourbaki, Algèbre Commutative ChI-VII, Paris: Hermann (1961-65). 
[ 6 ] J. F. Boutot, Schéma de Picard local, Lect. Notes Math., 632, Berlin, Heidelberg, New York: Springer (1978).

[ 7 ] M. P. Cavaliere and G. Niesi, On Serre's conditions in the form ring of an ideal, J. Math. Kyoto Univ., 21 (1981), 537-546.

[ 8 ] V. I. Danilov, The group of ideal classes of a completed ring, Math. USSR Sb., 6 (1968), 493-500.

[ 9 ] — On a conjecture of Samuel, Math. USSR Sb., 10 (1970), 127-137.

[10] _ Rings with a discrete group of divisor classes, Math. USSR Sb., 12 (1970), $368-386$.

[11] D. K. Faddeev, Group of divisor classes on the curve defined by the equation $\mathrm{X}^{4}+\mathrm{Y}^{4}=1$, Sov. Math. Dokl., I (1961), 1149-1151.

[12] H. Flenner, Divisorenklassengruppen quasihomogener Singularitäten, J. Reine Angew. Math., 238 (1981), 128-160.

[13] R. Fossum, The divisor class group of a Krull domain, Berlin-Heidelberg-New York: Springer (1973).

[14] S. Greco and P. Salmon, Topics in m-adic Topologies, Berlin-Heidelberg-New York: Springer (1971).

[15] A. Grothendieck, Cohomologie locale des faisceaux cohérents et Théorèmes de Lefschetz locaux et globaux (SGA 2), Amsterdam: North Holland (1968).

[16] A. Grothendieck et J. Dieudonné, Eléments de géométrie algébrique. Ch. III; Ch. IV (Quartième Partie), Publ. Math. Inst. Hautes Etud. Sci., 11;32 (1961; 1967).

[17] J. Lipman, Rational Singularities with applications to algebraic surfaces and unique factorization, Publ. Math. Inst. Hautes Etud. Sci., 36 (1969).

[18] — Rings with discrete divisor class group: theorem of Danilov-Samuel, Am. J. Math., 101 (1979), 203-211.

[19] J. Matijevich, Three local conditions on a graded ring, Trans. Am. Math. Soc., 205 (1975), 275-284.

[20] D. Portelli e W. Spangher, Condizioni di fattorialità ed anello graduato associato ad un ideale, Ann. Univ. Ferrara Nuova Ser. Sez. VII, 28 (1982), 181-195.

[21] —, On the divisor class group of localizations, completions and Veronesean subrings of $Z$-graded Krull domains, Ann. Univ. Ferrara Nuova Ser. Sez. VII, 30 (1984), 97-118.

[22] L. J. Jr. Ratliff, On Rees localities and $H_{i}$-local rings, Pac. J. Math., 60 (1975), 169-194.

[23] L. Robbiano and G. Valla, Primary powers of a prime ideal, Pac. J. Math., 63 (1976), 491-498.

[24] M. E. Rossi, Altezza e dimensione nell'anello graduato associato ad un ideale, Rend. Semin. Mat. Torino, 36 (1977-78), 305-312.

[25] P. Salmon, Su un problema posto da P. Samuel, Atti Accad. Naz. Lincei VIII Ser. Rend. Cl. Sci. Fis. Mat. Nat., 40 (1966), 801-803.

[26] P. Samuel, On unique factorization domains, Ill. J. Math., 5 (1961), 1-17.

[27] —- Sur les anneaux factoriels, Bull. Soc. Math. Fr., 89 (1961), 155-173.

[28] G. Scheja, Einige beispiele faktorieller lokaler Ringe, Math. Ann., 172 (1967), $124-134$.

[29] R. Y. Sharp, Local cohomology theory in commutative algebra, Q. J. Math. Oxf. II Ser., 21 (1970), 425-434.

[30] - Some results on the vanishing of local cohomology modules, Proc. Lond. Math. Soc. III Ser., 30 (1975), 177-195.

[31] S. Yuan, Reflexive modules and Algebra Class group over noetherian integrallyclosed domains, J. Algebra, 32 (1974), 405-417. 
Dipartimento di Scienze matematiche

Università degli Studi di Trieste

Piazzale Europa n. 1

$34100-T R I E S T E$

Italy 